\title{
Quantum dynamics of attosecond electron pulse compression
}

\author{
Peter Baum ${ }^{1,2, a)}$ \\ ${ }^{1}$ Ludwig-Maximilians-Universität München, Am Coulombwall 1, 85748 Garching, Germany \\ ${ }^{2}$ Max-Planck-Institute of Quantum Optics, Hans-Kopfermann-Str. 1, 85748 Garching, Germany
}

(Received 28 September 2017; accepted 15 November 2017; published online 12 December 2017)

\begin{abstract}
If an electron beam is periodically modulated in velocity, for example by laser field cycles, it can transform upon further propagation into a train of attosecond or shorter electron pulses. Here, I investigate the quantum mechanics of such an approach by numerically solving the Schrödinger equation in the time domain. There is a limit for the shortest electron pulses that can be achieved, and it depends on simple relations between the electron energy, the laser period, and the modulation strength. These results allow to design future experiments and to compare the measured electron pulse shapes to their quantum limit. Published by AIP Publishing. https://doi.org/10.1063/1.5006864
\end{abstract}

\section{INTRODUCTION}

Science and technology are continuously improving the spatial and temporal resolution of all kinds of imaging approaches, in order to directly visualize fundamental structures and processes on their natural length and time scales. A recent contribution from our laboratory has been the unification of electron microscopy/diffraction with attosecond time resolution, ${ }^{1}$ which brings together the space-time regimes of atoms and light-cycles. The key concept is a periodic modulation of an electron beam's forward momentum by the optical cycles of laser light. ${ }^{2-6}$ Figure 1 illustrates this basic idea, which allows producing attosecond electron pulses, ${ }^{7-9}$ but which is also useful for many other applications, for example in synchrotrons, ${ }^{10}$ free-electron lasers, ${ }^{11,12}$ for quantum optics with free electrons, ${ }^{5,9,13}$ laser-particle acceleration, ${ }^{14}$ terahertz-control of electrons, ${ }^{6,15,16}$ or for attosecond electron microscopy and diffraction. ${ }^{1}$ A femtosecond (or longer) electron pulse (blue) passes through a beam modulation element (green) that is driven by the optical cycles of a laser wave (red). The electron beam is assumed to have subrelativistic energy (typically $30-300 \mathrm{keV}$ in ultrafast electron microscopy and diffraction), so that the vacuum is dispersive. Alternatively, a dedicated dispersion section can be applied. If the laser-electron interaction at the beam modulator is formed such that the laser's electromagnetic fields create some additional electron momentum in the forward/ backward direction, the electron beam obtains a timedependent velocity modulation, i.e., a sequence of faster and slower parts in dependence on the arrival time at the interaction region [yellow line in Fig. 1(b)]. After further propagation in free space [black arrows in Fig. 1(b)], this momentum modulation gradually converts the beam into a train of ultrashort pulses [red line in Fig. 1(c)]. Afterwards, the pulses over-compress and disperse again [violet line in Fig. 1(c)]. In practice, the time-dependent momentum gain and loss can, for example, be realized with microwave cavities, ${ }^{17-19}$ with ponderomotive optical gratings in free space, ${ }^{3,20,21}$ with terahertz fields, ${ }^{6,15,16}$ or with laser pulses on nanostructures, ${ }^{5,22}$ sub-wavelength arrays, ${ }^{7,8}$ or graphite flakes. ${ }^{9}$ If

a)peter.baum@1mu.de long electron pulses and long laser pulses are applied, a train of attosecond electron pulses emerges ${ }^{7}$ that is synchronized to the laser field cycles ${ }^{3,9,20}$ in a way that allows pump-probe attosecond diffraction and microscopy of cycle-reversible phenomena. ${ }^{1}$

\section{APPROACH AND RESULTS}

Here, I report on the quantum dynamics of laser-based electron pulse compression and in particular, on the temporal quantum limits that the final pulses must obey. To this end, classical Monte-Carlo simulations with point particles are compared to a one-dimensional numerical solution of the Schrödinger equation in the time domain. In some implied way, this approach is related to earlier considerations of laser-electron interaction via photon sidebands in the energy domain, ${ }^{23-26}$ a measurement of which allows concluding back to the time domain via quantum-state-reconstruction. ${ }^{9}$ The initial conditions are electrons with an energy $E_{0}$ and velocity $v_{0}$, for example, $E_{0}=70 \mathrm{keV}$ and $v_{0}=0.476 c ; c$ is the speed of light. The laser has a wavelength $\lambda_{\text {laser }}$. The incoming electron pulses are assumed to have hundreds-offemtosecond durations ${ }^{27}$ and a temporal/longitudinal coherence length ${ }^{28} \xi_{t}$, which is longer than the laser oscillation period $T_{\text {laser }}=\lambda_{\text {laser }} / c$, for example $\xi_{t}>8$ fs at $0.2 \mathrm{eV}$ energy bandwidth vs. $T_{\text {laser }}=3.45 \mathrm{fs}$ at $\lambda_{\text {laser }}=1030 \mathrm{~nm}$. Hence, the initial electron wave function covers more than one laser cycle coherently, ${ }^{27}$ which is a prerequisite for proper compression. ${ }^{3}$ For chirped/pre-dispersed incoming electron pulses, ${ }^{27}$ the relevant coherence is the local/instantaneous coherence time, which is given by the instantaneous uncorrelated energy spread and which is therefore longer than the $\xi_{t}$ derived above, which is a lower limit. ${ }^{28}$ The beam modulation element is supposed to cause a direct transfer of the laser's sinusoidal field oscillations into a sinusoidal momentum change of the electron beam in time. We treat here the case of long electron pulses and long laser pulses [see Fig. 1(a)]; the effects of single-cycle modulation will be discussed below. Potential phase shifts from modulator resonances ${ }^{6}$ or time-integrated interactions at metal foils ${ }^{23,27}$ are irrelevant for laser pulses with a sufficiently large number of cycles. Propagation effects within the modulation element are also neglected, that is the 


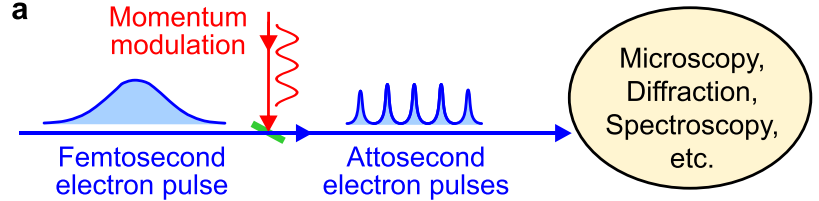

b
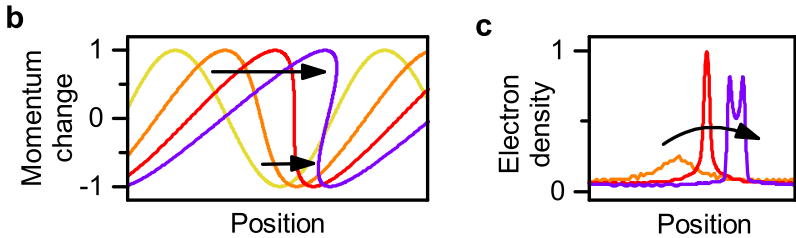

FIG. 1. Attosecond electron pulse formation by optical-cycle modulation; see Ref. 3. (a) Basic concept: a long electron pulse (blue) passes by a beam modulation element (green) that is driven by the optical cycles of laser light (red). The periodic acceleration and deceleration transform the pulse into a train of attosecond sub-pulses, which can be used for various space-timeimaging applications (yellow circle); see Ref. 1. (b) Illustration in the momentum domain. Initially, there is a sinusoidal momentum modulation (yellow). During further pulse propagation (black arrows), the electron dispersion lets higher-energetic parts catch up with lower-energetic ones (orange) toward a best-possible compression (red), followed by overcompression (violet). (c) Illustration in the time domain. Over time (black arrows), the instantaneous electron density peaks up (red) and afterwards spreads out again (violet).

electrons pass through the modulator within a time that is much shorter than half a laser oscillation cycle ${ }^{6,27}$ or in a phase-matched/quasi-phase-matched way., ${ }^{7,8}$ The mean velocity $v_{0}$ is modulated in time with a peak amplitude $\Delta v_{\max }$ (defined in the laboratory frame) that characterizes the compression strength and defines the distance to the temporal focus, i.e., the point at which the electron pulses reach a minimum duration. It is further assumed that the temporal focus is farther away from the laser interaction point than several laser wavelengths, i.e., the induced electron momentum changes are low compared to the central momentum, $\Delta v_{\max } \ll v_{0}$. In other words, the pulses should not substantially reshape while travelling through the beam modulator or through the temporal focus. The discussion is also restricted to the physics on the main axis, i.e., to one dimension, which is justifiable for collimated electron beams in velocity-matched geometries ${ }^{6,27}$ without magnetic fields ${ }^{29}$ or for a beam that is much smaller than the modulating nearfields. ${ }^{5}$ Although most of the relativistic effects are accounted for, the results are intended for electron energies below the $\mathrm{MeV}$ regime. Overall, these approximations seem well fulfilled in all the experimental attosecond approaches mentioned above.

A useful parameter for the following laboratory-frame considerations is the compression strength around the modulation's zero crossings in velocity-change-per-time units, given by $g_{v}=2 \pi c \Delta v_{\max } / \lambda_{\text {laser }}=2 \pi \Delta v_{\max } / T_{\text {laser }}$. To the first order, the temporal focus can be expected ${ }^{30}$ at a distance $L_{\text {focus }}$, which is given by

$$
L_{\text {focus }}=\frac{v_{0}^{2}}{g_{v}}=\frac{v_{0}^{2} T_{\text {laser }}}{2 \pi \Delta v_{\max }} .
$$

Shorter oscillation periods, for example, high-frequency microwaves $^{30}$ or-in this work-optical laser cycles, are therefore beneficial for achieving a nearby temporal focus. A closer focus often means shorter pulses, as we will see.

Figure 2(a) shows the results of a Monte-Carlo simulation with 13000 particles per femtosecond as a homogeneous distribution in time. The example conditions are $\lambda_{\text {laser }}$ $=1030 \mathrm{~nm},{ }^{31} E_{0}=70 \mathrm{keV}, v_{0}=0.476 \mathrm{c}, \Delta v_{\max }= \pm 6000 \mathrm{~m} / \mathrm{s}$, $\xi_{t}=8 \mathrm{fs}$, and $T_{\text {laser }}=3.45 \mathrm{fs}$. The point-particle electrons have a random uncorrelated energy spread of $0.2 \mathrm{eV}$ (full width at half maximum), which is a realistic assumption. ${ }^{27,32}$ The corresponding velocity spread of $\sim 150 \mathrm{~m} / \mathrm{s}$ is more than 40 times lower than $\Delta v_{\max }$ and hence largely irrelevant for the results. The colors denote the time-dependent electron density for increasing propagation times ( $t_{\text {prop }}$, picosecond range) that include the temporal focus (dotted line). In the pulse-shape domain ( $t_{\text {pulse }}$, femtosecond range), the plot is restricted to the central region around a single attosecond peak; there are, of course, multiple such compressions happening in each of the laser's multiple cycles; compare Fig. 1(a). The initially homogeneous distribution at $t_{\text {prop }}=0$ gradually converts to a singularity at the temporal focus, followed by over-compression with the typical side peaks, ${ }^{6,30}$ which are a consequence of the highest probability of maximum/minimum electron modulation at the turning points (cusps) of the sinusoidal acceleration/deceleration; compare Figs. 1(b) and 1(c). The minimum pulse duration at the temporal focus is $\sim 15$ as, which is unrealistic, as we will see.

Figure 2(b) shows the compression dynamics if the quantum mechanics of the electron is accounted for. The CrankNicolson algorithm ${ }^{33}$ was used to numerically solve the Schrödinger equation of an initial electron wave function with the above mentioned parameters. To simplify the computation, the problem is first taken to the rest frame of the electron and after the quantum simulations back to the laboratory frame by Lorentz transformations. The modulation length in the rest frame becomes $\gamma v_{0} T_{\text {laser }}$ (the packet sweeps with sublight speed over the 3.45 -fs-modulation) and the peak velocity gain in the rest frame becomes $\Delta v_{\text {rest }} \approx \gamma^{2} \Delta v_{\max }$ via relativistic velocity addition at $\Delta v_{\max } \ll v_{0}$. The Crank Nicholson algorithm uses a length step of $1.95 \mathrm{~nm}$ and a time step $\left(t_{\text {prop }}\right)$ of $0.25 \mathrm{ps}$. After the simulation, the laboratory frame dimensions, $t_{\text {pulse }}$ and $t_{\text {prop }}$, are obtained from the proper-time and length steps via Lorentz back-transformations.

The colors in Fig. 2(b) denote again the time-dependent electron density of the pulses (that is square of the wave function, $|\psi|^{2}$ ) during propagation through the temporal focus. There are four immediate observations. Before the temporal focus, the compression dynamics is very similar to the classical case. At the temporal focus (dotted line), the pulses are longer and have a finite duration $\tau_{\text {focus }}$. After the temporal focus, there is a lot of interference and the pulse splits into sub-pulses. In the very end of the simulated range (above 45 ps), one can see parts of the adjacent pulses in the pulse train coming in from earlier and later times, i.e., from left and right in Fig. 2(b), causing additional interferences. Figure 2(c) shows the squared electron wave function $|\psi|^{2}$ at selected times before, slightly after and far after the temporal focus. One can see the narrowing of the main peak (black arrows) to a pulse duration of 228 as (full-width-at-half-maximum), but there is also a more or less constant background 

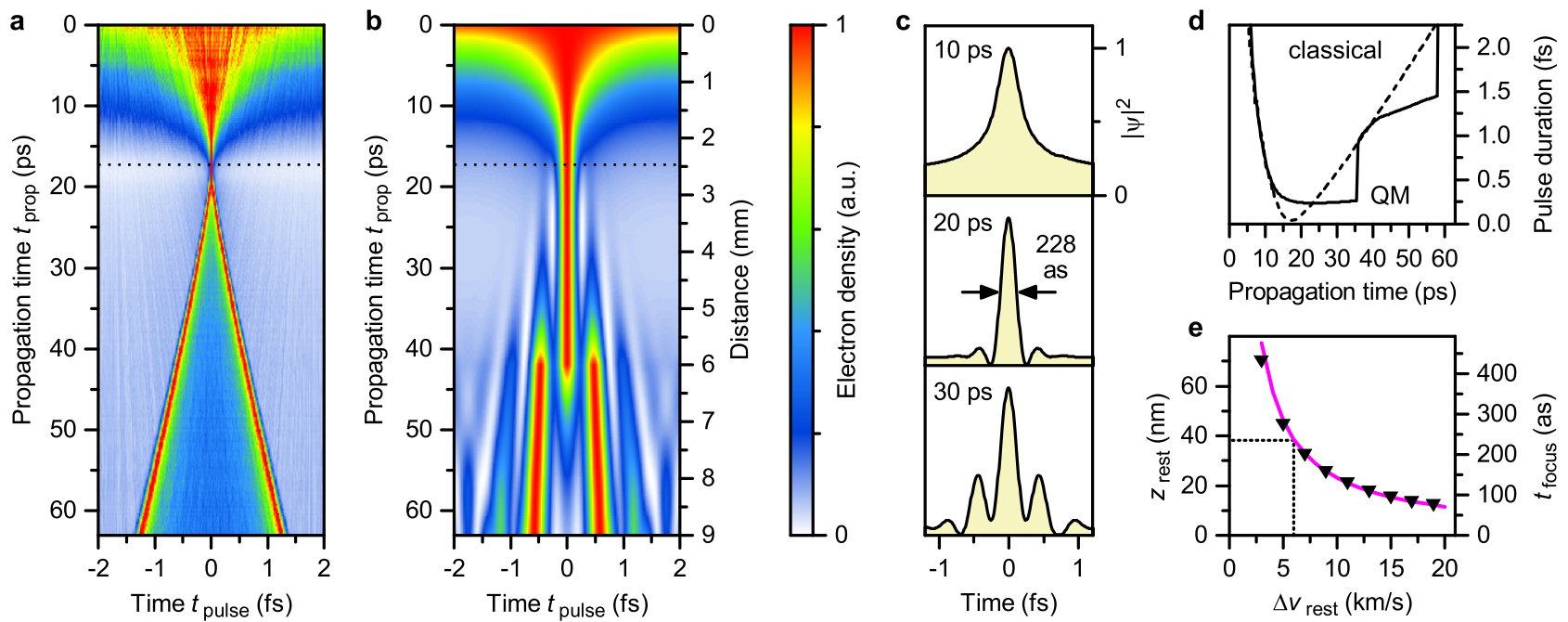

FIG. 2. Quantum dynamics of attosecond electron pulse compression. (a) Electron pulse shape $\left(t_{\text {pulse }}\right.$-scale) over propagation time after modulation $\left(t_{\text {prop }}\right)$ if assuming classical mechanics (point particles). (b) Electron pulse shape with quantum-mechanical effects. The dotted line denotes the location of the temporal focus. (c) Three snapshots of the quantum-mechanical wave function $\left|\psi^{2}\right|$ shortly after compression (upper panel), slightly after the temporal focus (middle panel), and somewhat later (lower panel). Evident are coherent interferences that cause the appearance of side lobes and also restrict the minimum pulse duration of the main peak. (d) Time evolution of the electron pulse duration (full-width-at-half-maximum) for classical particles (dashed line) and the quantummechanical case (solid line). (e) Shortest-possible quantum-mechanical electron pulse length $z_{\text {rest }}$ (rest frame) and duration $\tau_{\text {focus }}$ (laboratory-frame) in dependence on the rest-frame modulation strength $\Delta v_{\text {rest }}$. A quantum limit can be derived by approximation (purple line). The dashed lines denote the conditions used in (a) and (b).

current for all times, which is initially large [upper panel in Fig. 2(c)], then gradually decreases towards the temporal focus [middle panel in Fig. 2(c)], and afterwards converts to interferences [lowest panel in Fig. 2(c)]. Such a background current has been predicted ${ }^{3}$ and observed; ${ }^{1,9}$ it originates from the temporal de-compression that is caused by every other laser-half cycle.

Figure 2(d) shows the evolution of the electron pulse duration during propagation for the cases of point particles (dashed line) and quantum wave functions (solid line). I define here the pulse duration as the full-width-at-half-maximum, which is the usual definition in laser spectroscopy, because it often corresponds quite well to practically achievable time resolutions at typical single-to-noise levels. The classical evolution of the pulse duration shows an almostzero pulse duration at $\sim 17 \mathrm{ps}$ after modulation. The following over-compression has a different rate due to the development of a double-lobe pattern in the over-compressed pulses [compare Fig. 2(a)]. The more realistic quantum-mechanical case [solid line in Fig. 2(d)] shows almost the same kind of compression dynamics in the initial stages. The temporal focus is located slightly after the classical focus; the shortest pulses have a finite duration of 228 as. Interestingly, this minimum duration is preserved for a substantially longer time range as compared to the classical case. This large depth-of-temporal-focus is a consequence of the quantum interferences, which tend to first raise a pair of side-pulses instead of lengthening the main lobe; see Fig. 2(b) and the middle panel of Fig. 2(c). Later, in the over-compression regime, the pulse duration increases abruptly in a stepwise way, as soon as new side peaks of the quantum interferences reach the chosen half-maximum threshold.

The rest-frame quantum simulations allow deriving some fundamental relations on the pulse duration. Figure 2(e) shows the minimum electron pulse length $z_{\text {rest }}$ (in the rest frame) at the temporal focus in dependence on the restframe peak velocity modulation $\Delta v_{\text {rest }} \approx \gamma^{2} \Delta v_{\max }$. The plotted time scale is for the laboratory frame at $70 \mathrm{keV}$. One finds that a stronger modulation produces shorter pulses [triangles in Fig. 2(e)]. Assuming an indirect proportionality of the shortest-possible electron pulse length $z_{\text {rest }}$ with $\Delta v_{\text {rest }}$, we obtain the purple fit curve and can extract a phenomenological equation $z_{\text {rest }} \approx 2 \hbar /\left(m_{e} \Delta v_{\text {rest }}\right)$. In the laboratory frame, we obtain a minimum pulse duration of

$$
\tau_{\text {focus }} \approx \frac{2 \hbar}{\gamma^{3} m_{e} v_{0} \Delta v_{\max }} .
$$

This result resembles the uncertainty principle, i.e., a stronger momentum width corresponds to a more localized wave function and hence shorter pulses. The fitted factor of $\sim 2$ instead of the usual 1/2 for Gaussian wave packets is a consequence of the applied pulse length definitions (full-width) and the complicated shape of the energy spectrum after sinusoidal modulation. ${ }^{25}$ Analytical time-domain calculations are in progress and will be reported elsewhere, but for now, Eq. (2) should be understood as an approximation. Interestingly, Eq. (2) is independent of any details of the modulation element and solely depends on the maximum velocity gain $\Delta v_{\max }$ that is practically achieved. It therefore provides a clear way of estimating the shortest electron pulses that can be expected from the given laser pulses and modulation concepts.

It may be interesting to express Eq. (2) in terms of the laser cycle period $T_{\text {laser }}$ and the location of the temporal focus, $L_{\text {focus }}$. One obtains

$$
\tau_{\text {focus }} \approx \frac{4 \pi \hbar L_{\text {focus }}}{\gamma^{3} m_{e} v_{0}^{3} T_{\text {laser }}} .
$$


At a given laser wavelength and electron energy, there is a direct linear relationship between the temporal focus distance and the achievable pulse duration. For example, if 500as pulses are sought for with $70-\mathrm{keV}$ electrons by using a 1030-nm laser, the temporal focus can be as far away as $4 \mathrm{~mm}$, like in our experiment. ${ }^{1}$ At the same conditions, sub100 -as pulses could only be expected at sub-mm distance. Higher electron energies and slower laser cycles are beneficial. For example, at $200 \mathrm{keV}$ and with a $2-\mu \mathrm{m}$ laser, sub100 -as pulses could be produced at a convenient $10-\mathrm{mm}$ distance.

\section{EXAMPLE DEPENDENCIES}

Figure 3 illustrates several such dependencies in more detail. Figure 3(a) shows how the temporal focus that can produce electron pulses of a given quantum-limited duration (100 as, 200 as or 300 as) moves farther away for higher-energy electrons due to their increased $v_{0}$. Figure 3(b) shows how short electron pulses can be expected for a given focal distance (often mechanically constrained in an experiment) and electron acceleration voltage (often also rather fixed). Figure 3(c) shows the influence of the laser wavelength. For a given focal distance $(2 \mathrm{~mm}, 4 \mathrm{~mm}$, or $6 \mathrm{~mm}$ ) and at the example energy of $70 \mathrm{keV}$, the quantum-limited electron pulses shorten substantially if longer laser wavelengths are used. Laser compression fields in the infrared or $\mathrm{THz}$ regime ${ }^{6}$ are therefore beneficial, provided that there is enough laser power available for generating the necessary modulation strength. The observations of Fig. 3(c) also explain why quantum effects are not substantial in the microwave regimes, where rather jitter/synchronization
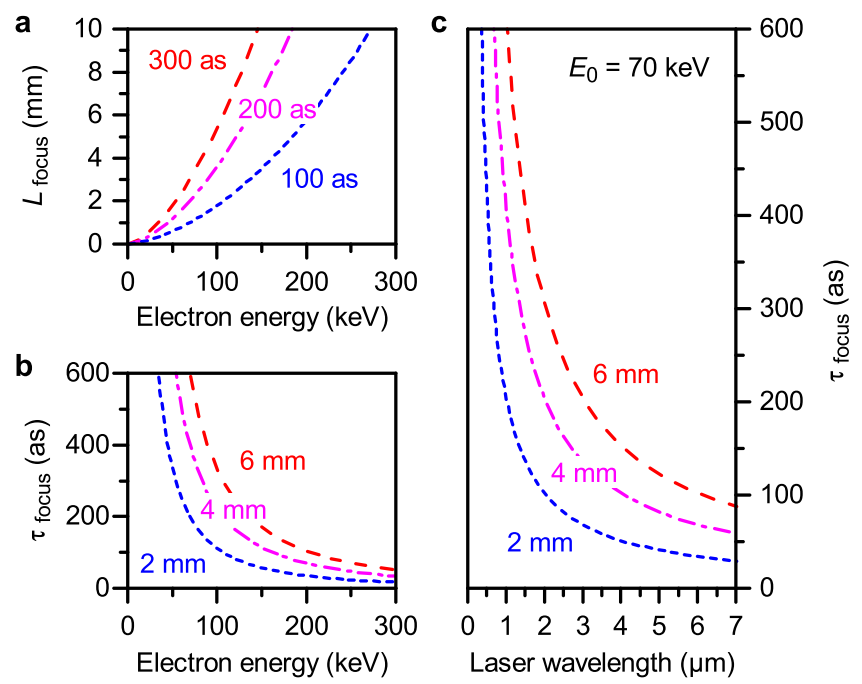

Laser wavelength $(\mu \mathrm{m})$

FIG. 3. Quantum-mechanical pulse duration limits in dependence on the electron energy and temporal focus location. (a) Focus position for three different quantum-limited electron pulse durations (blue/dashed, 100 as; purple/dash-dot, 200 as; red/long dash, 300 as) in dependence on the electron energy. The modulation wavelength is $1030 \mathrm{~nm}$. (b) Quantum-limited pulse duration for three different locations of the temporal focus (blue/dashed, $2 \mathrm{~mm}$; purple/dash-dot, $4 \mathrm{~mm}$; red/long dash, $6 \mathrm{~mm}$ ) in dependence on the electron energy. The modulation wavelength is $1030 \mathrm{~nm}$. (c) Quantumlimited pulse duration at $70 \mathrm{keV}$ electron energy for three different locations of the temporal focus (blue/dashed, $2 \mathrm{~mm}$; purple/dash-dot, $4 \mathrm{~mm}$; red/long dash, $6 \mathrm{~mm}$ ) in dependence on the optical modulation wavelength. Longer laser wavelengths in the infrared make substantially shorter pulses at a given spot, provided that there is enough optical power available. issues or space charge effects restrict the achievable compression, ${ }^{19,34,35}$ and not the fundamental limits. The quantum limit of Eq. (3) may, however, not entirely be irrelevant for $\mathrm{THz}$ compressors with cm-range focal distances. ${ }^{6}$ For example, at $E_{0}=70 \mathrm{keV}$, with $T_{\text {laser }} \approx(0.3 \mathrm{THz})^{-1}$ and $L_{\text {focus }} \approx 50 \mathrm{~cm}$, which are roughly the conditions of Ref. 6 , we obtain $\tau_{\text {focus }} \approx 50$ as. The relativistic energies of synchrotrons and free-electron lasers promise in principle even shorter pulses, ${ }^{4}$ but applicability of such sources to atomic-scale imaging and diffraction is restricted by the too short de Broglie wavelength.

\section{CHIRP AND ISOLATED PULSES}

The discussion so far has neglected another effect that can become substantial if the input electron pulses before the compression are chirped. Such a time-energy correlation is natural in laser-triggered electron sources due to some unavoidable conversion of the emission's energy spread into dispersion. ${ }^{36}$ Space charge effects come on top and dense multi-electron electron pulses can develop a very clear and strong time-energy correlation. ${ }^{37}$ Such long pulses may locally still be monochromatic enough to have a good enough temporal coherence to cover more than one optical cycle, in order to produce efficient attosecond compression, but there will be a time-dependent dispersion of the central energy, and therefore, a time-dependent variation of the arrival time at a given target location. In order words, every pulse in the pulse train might individually compress rather well, according to the above simulations, but there might no longer be a temporal synchronization to the laser cycles that are necessary in a pump-probe experiment, ${ }^{1}$ for example for exciting electron dynamics in materials ${ }^{38-40}$ or metamaterials. ${ }^{41}$ Fortunately, the solution seems rather easy, namely with laser pulses of different chirp in the compression and excitation stages, designed such that the optical chirp difference matches the arrival time dispersion of the electron pulse train.

Isolated attosecond electron pulses may be needed for investigating certain dynamics that is not reversible between adjacent laser cycles, that is processes with time constants longer than $T_{\text {laser }}$. We mentioned several ideas for generating isolated attosecond pulses in our recent contribution ${ }^{1}$ and are working on their realization, for example via multi-stage compression. $^{42}$ I expect that the temporal compression of electron pulses by a single-cycle laser should to a large extent, at least approximately, retain the above derived quantum limits, especially Eq. (2), because the slope and curvature of a single-cycle laser modulation around the zero crossing is not far different from the shape of a true sinusoidal half-cycle. Multi-color fields ${ }^{13}$ that linearize the modulation slope can help in producing shorter pulses, but the simulations show that the quantum limit of Eq. (2) remains roughly valid even for a saw-tooth modulation.

\section{OUTLOOK}

In the long run, attosecond electron pulses, or pulse trains, promise a direct access to light-driven electronic motion in materials and nanostructures, in order to visualize 
the spatiotemporal electronic motions that define lightmatter interactions on a fundamental level. ${ }^{42}$ The above reported simulations of electron pulse compression by sinusoidal laser modulation show that quantum effects play a major role for the form and final duration of the electron pulses at the temporal focus. The reported equations will allow to design future experiments for the required temporal resolution and to compare the measured pulse shapes to the fundamental limit.

\section{ACKNOWLEDGMENTS}

This work was supported by the European Research Council (grant DIVI) and the Munich-Centre for Advanced Photonics. I thank Maxim Tsarev for the assistance with special relativity, Yuya Morimoto and Dominik Ehberger for the inspiring discussions, and Ferenc Krausz for the awesome general support.

${ }^{1}$ Y. Morimoto and P. Baum, "Diffraction and microscopy with attosecond electron pulse trains," Nat. Phys. (2017).

${ }^{2}$ A. A. Zholents and M. S. Zolotorev, "Femtosecond x-ray pulses of synchrotron radiation," Phys. Rev. Lett. 76(6), 912-915 (1996).

${ }^{3}$ P. Baum and A. H. Zewail, "Attosecond electron pulses for 4D diffraction and microscopy,” Proc. Natl. Acad. Sci. U.S.A. 104(47), 18409-18414 (2007).

${ }^{4}$ E. Hemsing, G. Stupakov, D. Xiang, and A. Zholents, "Beam by design: Laser manipulation of electrons in modern accelerators," Rev. Mod. Phys. 86(3), 897-941 (2014).

${ }^{5}$ A. Feist, K. E. Echternkamp, J. Schauss, S. V. Yalunin, S. Schaefer, and C. Ropers, "Quantum coherent optical phase modulation in an ultrafast transmission electron microscope," Nature 521(7551), 200 (2015).

${ }^{6}$ C. Kealhofer, W. Schneider, D. Ehberger, A. Ryabov, F. Krausz, and P. Baum, "All-optical control and metrology of electron pulses," Science 352(6284), 429-433 (2016).

${ }^{7}$ C. M. S. Sears, E. Colby, R. Ischebeck, C. McGuinness, J. Nelson, R. Noble, R. H. Siemann, J. Spencer, D. Walz, T. Plettner, and R. L. Byer, "Production and characterization of attosecond electron bunch trains," Phys. Rev. Spec. Top.-Accel. Beams 11(6), 061301 (2008).

${ }^{8}$ M. Kozak, J. McNeur, K. J. Leedle, H. Deng, N. Schonenberger, A. Ruehl, I. Hartl, J. S. Harris, R. L. Byer, and P. Hommelhoff, "Optical gating and streaking of free electrons with sub-optical cycle precision," Nat. Commun. 8, 14342 (2017).

${ }^{9}$ K. E. Priebe, C. Rathje, S. V. Yalunin, T. Hohage, A. Feist, S. Schaefer, and C. Ropers, "Attosecond electron pulse trains and quantum state reconstruction in ultrafast transmission electron microscopy," Nat. Photonics (to be published); e-print arXiv:1703.10589.

${ }^{10}$ R. W. Schoenlein, S. Chattopadhyay, H. H. W. Chong, T. E. Glover, P. A. Heimann, C. V. Shank, A. A. Zholents, and M. S. Zolotorev, "Generation of femtosecond pulses of synchrotron radiation," Science 287(5461), 2237-2240 (2000).

${ }^{11}$ A. A. Zholents and W. M. Fawley, "Proposal for intense attosecond radiation from an X-ray free-electron laser," Phys. Rev. Lett. 92(22), 224801 (2004).

${ }^{12}$ B. Garcia, E. Hemsing, T. Raubenheimer, L. T. Campbell, and B. W. J. McNeil, "Method to generate a pulse train of few-cycle coherent radiation," Phys. Rev. Accel. Beams 19(9), 090701 (2016).

${ }^{13}$ K. E. Echternkamp, A. Feist, S. Schafer, and C. Ropers, "Ramsey-type phase control of free-electron beams," Nat. Phys. 12(11), 1000 (2016).

${ }^{14}$ R. J. England, R. J. Noble, K. Bane, D. H. Dowell, C.-K. Ng, J. E. Spencer, S. Tantawi, Z. Wu, R. L. Byer, E. Peralta, K. Soong, C.-M. Chang, B. Montazeri, S. J. Wolf, B. Cowan, J. Dawson, W. Gai, P. Hommelhoff, Y.-C. Huang, C. Jing, C. McGuinness, R. B. Palmer, B. Naranjo, J. Rosenzweig, G. Travish, A. Mizrahi, L. Schachter, C. Sears, G. R. Werner, and R. B. Yoder, "Dielectric laser accelerators," Rev. Mod. Phys. 86(4), 1337-1389 (2014).

${ }^{15}$ L. Wimmer, G. Herink, D. R. Solli, S. V. Yalunin, K. E. Echternkamp, and C. Ropers, "Terahertz control of nanotip photoemission," Nat. Phys. 10(6), 432-436 (2014).
${ }^{16}$ E. A. Nanni, W. Q. R. Huang, K. H. Hong, K. Ravi, A. Fallahi, G. Moriena, R. J. D. Miller, and F. X. Kartner, "Terahertz-driven linear electron acceleration," Nat. Commun. 6, 8486 (2015).

${ }^{17}$ E. Fill, L. Veisz, A. Apolonski, and F. Krausz, "Sub-fs electron pulses for ultrafast electron diffraction," New J. Phys. 8, 272 (2006).

${ }^{18}$ L. Veisz, G. Kurkin, K. Chernov, V. Tarnetsky, A. Apolonski, F. Krausz, and E. Fill, "Hybrid DC-AC electron gun for fs-electron pulse generation," New J. Phys. 9, 451 (2007).

${ }^{19}$ T. van Oudheusden, P. L. E. M. Pasmans, S. B. van der Geer, M. J. de Loos, M. J. van der Wiel, and O. J. Luiten, "Compression of subrelativistic space-charge-dominated electron bunches for single-shot femtosecond electron diffraction," Phys. Rev. Lett. 105(26), 264801 (2010).

${ }^{20} \mathrm{P}$. Baum and A. H. Zewail, "4D attosecond imaging with free electrons: Diffraction methods and potential applications," Chem. Phys. 366(1-3), 2-8 (2009).

${ }^{21}$ M. Kozak, M. k, T. Eckstein, N. Schonenberger, and P. Hommelhoff, "Inelastic ponderomotive scattering of electrons at a high-intensity optical travelling wave in vacuum," Nat. Phys. (2017).

${ }^{22}$ M. Kruger, M. Schenk, and P. Hommelhoff, "Attosecond control of electrons emitted from a nanoscale metal tip," Nature 475(7354), 78-81 (2011).

${ }^{23}$ P. Reckenthaeler, M. Centurion, V. S. Yakovlev, M. Lezius, F. Krausz, and E. E. Fill, "Proposed method for measuring the duration of electron pulses by attosecond streaking," Phys. Rev. A 77(4), 042902 (2008).

${ }^{24}$ F. J. G. de Abajo and M. Kociak, "Electron energy-gain spectroscopy," New J. Phys. 10, 073035 (2008).

${ }^{25}$ B. Barwick, D. J. Flannigan, and A. H. Zewail, "Photon-induced near-field electron microscopy," Nature 462(7275), 902-906 (2009).

${ }^{26}$ S. T. Park, M. M. Lin, and A. H. Zewail, "Photon-induced near-field electron microscopy (PINEM): Theoretical and experimental," New J. Phys. 12, 123028 (2010).

${ }^{27}$ F. O. Kirchner, A. Gliserin, F. Krausz, and P. Baum, "Laser streaking of free electrons at $25 \mathrm{keV}$," Nat. Photonics 8(1), 52-57 (2014).

${ }^{28} \mathrm{P}$. Baum, "On the physics of ultrashort single-electron pulses for timeresolved microscopy and diffraction," Chem. Phys. 423, 55-61 (2013).

${ }^{29} \mathrm{C}$. Weninger and P. Baum, "Temporal distortions in magnetic lenses," Ultramicroscopy 113, 145-151 (2012).

${ }^{30}$ A. Gliserin, A. Apolonski, F. Krausz, and P. Baum, "Compression of single-electron pulses with a microwave cavity,” New J. Phys. 14, 073055 (2012).

${ }^{31}$ W. Schneider, A. Ryabov, C. Lombosi, T. Metzger, Z. Major, J. A. Fulop, and P. Baum, "800-fs, 330- $\mu \mathrm{J}$ pulses from a 100-W regenerative Yb:YAG thin-disk amplifier at $300 \mathrm{kHz}$ and $\mathrm{THz}$ generation in $\mathrm{LiNbO}_{3}$," Opt. Lett. 39(23), 6604-6607 (2014).

${ }^{32}$ R. Bormann, S. Strauch, S. Schaefer, and C. Ropers, "An ultrafast electron microscope gun driven by two-photon photoemission from a nanotip cathode," J. Appl. Phys. 118(17), 173105 (2015).

${ }^{33}$ J. Crank and P. Nicolson, "A practical method for numerical evaluation of solutions of partial differential equations of the heat-conduction type," Adv. Comput. Math. 6(3-4), 207-226 (1996).

${ }^{34}$ A. Gliserin, M. Walbran, F. Krausz, and P. Baum, "Sub-phonon-period compression of electron pulses for atomic diffraction," Nat. Commun. 6, 8723 (2015)

${ }^{35}$ M. Walbran, A. Gliserin, K. Jung, J. Kim, and P. Baum, "5-femtosecond laser-electron synchronization for pump-probe crystallography and diffraction," Phys. Rev. Appl. 4(4), 044013 (2015).

${ }^{36}$ M. Aidelsburger, F. O. Kirchner, F. Krausz, and P. Baum, "Single-electron pulses for ultrafast diffraction," Proc. Natl. Acad. Sci. U.S.A. 107(46), 19714-19719 (2010).

${ }^{37}$ B. J. Siwick, J. R. Dwyer, R. E. Jordan, and R. J. D. Miller, "Ultrafast electron optics: Propagation dynamics of femtosecond electron packets," J. Appl. Phys. 92(3), 1643-1648 (2002).

${ }^{38}$ V. S. Yakovlev, M. I. Stockman, F. Krausz, and P. Baum, "Atomic-scale diffractive imaging of sub-cycle electron dynamics in condensed matter," Sci. Rep. 5, 14581 (2015).

${ }^{39}$ J. Stingl, F. Zamponi, B. Freyer, M. Woerner, T. Elsaesser, and A. Borgschulte, "Electron transfer in a virtual quantum state of $\mathrm{LiBH}_{4}$ induced by strong optical fields and mapped by femtosecond $\mathrm{x}$-ray diffraction," Phys. Rev. Lett. 109(14), 147402 (2012).

${ }^{40}$ H. C. Shao and A. F. Starace, "Energy-resolved coherent diffraction from laser-driven electronic motion in atoms," Phys. Rev. A 96(4), 042706 (2017).

${ }^{41}$ A. Ryabov and P. Baum, "Electron microscopy of electromagnetic waveforms," Science 353(6297), 374-377 (2016).

${ }^{42} \mathrm{P}$. Baum and F. Krausz, "Capturing atomic-scale carrier dynamics with electrons," Chem. Phys. Lett. 683, 57-61 (2017). 